\title{
Vasectomy Occlusion Technique Combining Thermal Cautery and Fascial Interposition
}

\author{
Michel Labrecque \\ Research Center, Hôpital Saint-François d'Assise, Centre Hospitalier Universitaire de Québec, \\ Québec, Canada
}

\begin{abstract}
Introduction: Recent research on vasectomy shows that combining cautery and fascial interposition (FI) achieves the most effective occlusion of the vas and minimizes the risk of failure. We present a technique that combines cautery and FI and is suitable for low-resource settings.

Surgical Technique: The surgical technique consists of 1) exposing the vas with the no-scalpel approach; 2) cauterizing the epithelium of lumen of the vas using a portable battery-powered cautery device; 3 ) performing FI by grasping internal spermatic fascia and applying a free tie with suture material on the fascia to cover the prostatic stump of the vas and separate the two ends of the cut vas; and 4) excising a small 0.5 to $1 \mathrm{~cm}$ of the testicular stump.

Comments: To maximize vasectomy effectiveness, vasectomy providers should consider learning thermal cautery and FI to occlude vas deferens.
\end{abstract}

Key words: vasectomy; vas deferens; cautery; urological surgery procedure; male; sterilization Int Braz J Urol. 2011; 37:630-635

\section{INTRODUCTION}

Recent developments in vasectomy have shown that: 1) the no-scalpel approach to expose the vas (known as the no-scalpel vasectomy, or NSV) reduces the risk of surgical complications over incisional techniques; 2) ligation of the vas and excision of a small segment between the ligatures (LE) is associated with an unacceptable risk of failure and should not be performed; 3 ) adding fascial interposition (FI) to LE reduces the risk of failure; and 4) cautery of the mucosa of the lumen of the vas combined with FI appears to achieve the most effective occlusion of the vas (1-3). Although NSV has been widely adopted (4,5), LE is still by far the most common vas occlusion technique performed in many parts of the world (5). Recently, the Program for Appropriate Technology in Health (PATH) recommended that FI and thermal cautery be introduced into existing and new vasectomy programs and that providers be trained in this method to maximize the cost-effectiveness of ongoing programs (6).

The surgical technique described below uses NSV (7) to expose the vas and the thermal mucosal cautery and FI occlusion technique to occlude the vas. This occlusion technique was originally described by Moss (8), who used metal clips to perform FI. It has since been performed with a very high rate of success in thousands of men $(9,10)$. In the technique described, the metal clip is replaced by a free tie on the vas sheath (see step 9 of the surgical technique). Although this adaptation of Moss's technique can be employed in any setting, it is particularly suitable for low-resource settings where silk thread is already commonly used to ligate the vas (5) and where metal clips or other options to perform FI -such as sterile suture material mounted with a needle (11)- are not available. This is the case in 
many Asian countries (5) and may also be the case in Africa and Latin America. The only material that the new technique needs, in addition to the instruments and material already required to perform NSV and LE, is a portable battery-powered thermal cautery device. This kind of device is available from various suppliers (e.g. www.ameditech.com, www.vasectomy.ca, www.instrumedindia.com) and is easier and much more convenient to use than an electrical cautery device because of its small size and the absence of wires.

\section{SURGICAL TECHNIQUE}

1. Expose the vas outside the scrotum using the NSV technique. The NSV technique is described in detail elsewhere (7).

2. Completely separate the sheath (internal spermatic fascia) from the exposed segment of the vas (Figure-1).

a. Tip: Make sure only a partial thickness of the vas is grasped - do not encircle the vas with the ring forceps - otherwise control of the vas will be lost when it is cut.

b. Tip: The length of the bare vas segment does not need to be as long as when using the LE technique. When stripping the sheath, open the blades of the dissecting forceps 3 to $5 \mathrm{~mm}$, i.e., less than half of the length used for LE.

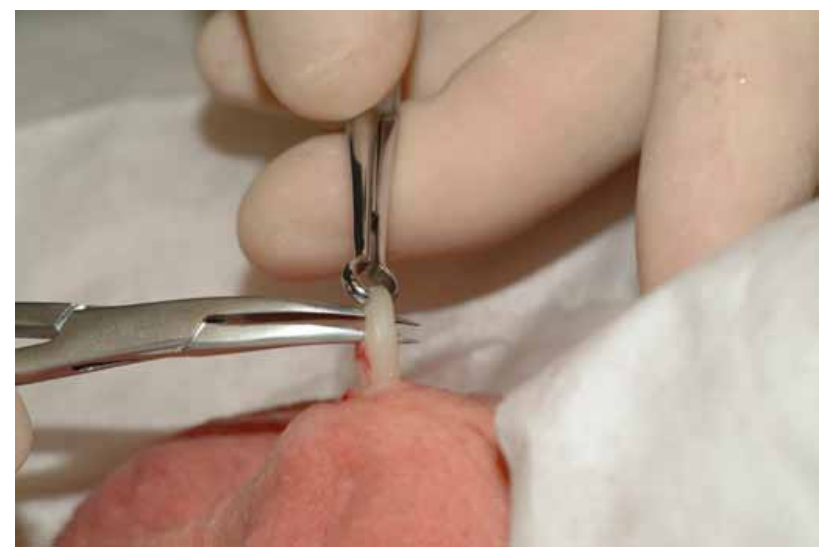

Figure 1 - Opening the dissecting forceps to strip the sheath.
3. Hemi-transect the prostatic end of the bare vas at mid-distance between the ring clamp teeth and the remaining vas sheath.

a. Tip: The cut must be deep enough to expose the lumen but the posterior wall of the vas should remain intact.

b. Tip: Hemi-transection may be performed with small sharp scissors or with the thermal cautery device (Figures 2 and 3 ).

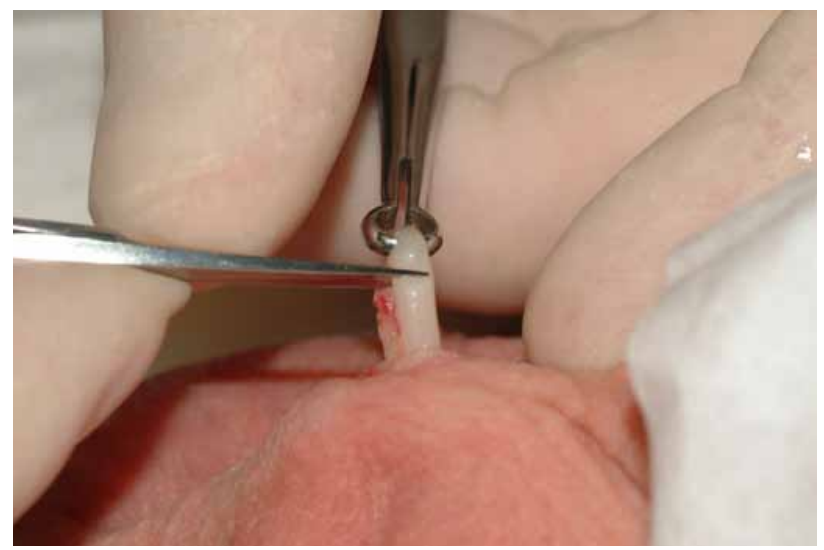

Figure 2 - Hemi-transecting the vas with scissors.

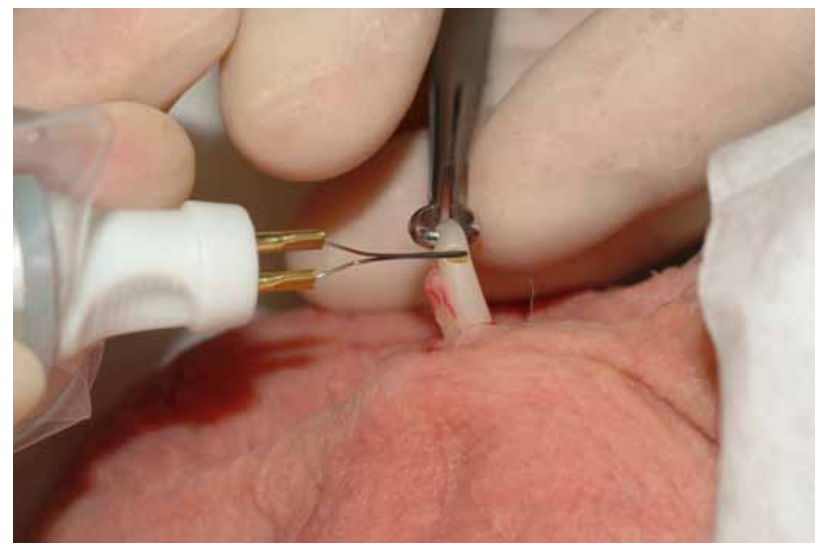

Figure 3 - Hemi-transecting the vas with a thermal cautery device.

4. Insert the cold cautery tip into the hemi-cut towards the lumen of the prostatic vas. Then bring it parallel to the vas while fully inserting the tip into the lumen. 
a. Tip: Verify that the device is functioning properly before starting the procedure. The tip must become red when the device is turned on.

b. Tip: Do not insert the tip into the lumen when the device is turned on. The tip should be cold when inserted.

c. Tip: Hold the device like a pencil and stabilize it using the thumb of the other hand.

d. Tip: Make sure the tip is inside and parallel to the lumen before turning on the device.

5. Turn on the cautery device for 2 to 3 seconds, until the vas just starts to become opaque or fumes appear (Figure-4).

a. Tip: Take care not to over-burn the vas. Only the epithelium must be destroyed: not the muscular vas wall.

b. Tip: Cautery time varies with battery strength. Cautery with brand-new alkaline batteries may only take 1 second.

c. Tip: The tip may stick into the vas. Gentle traction on the tip with a rotating movement should help deliver the tip from the lumen. Turning on the device for another second while pulling the device out may help. Again, take care not to burn the vas excessively.

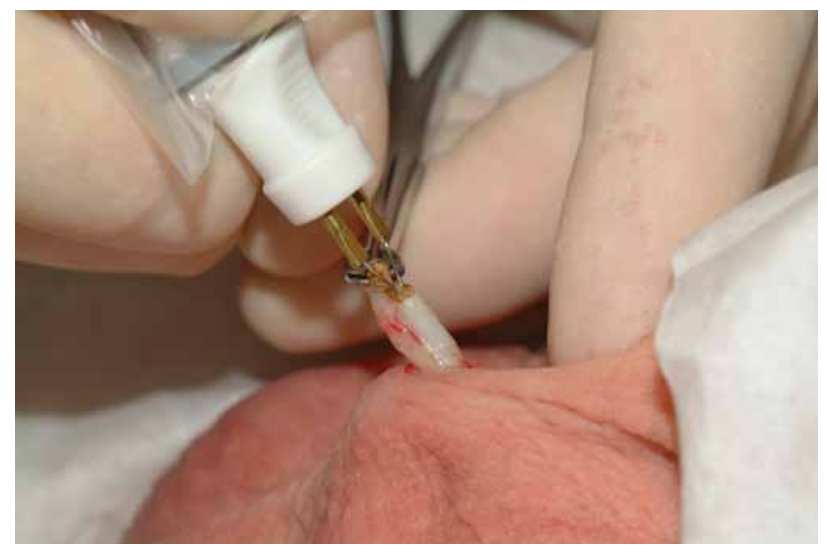

Figure 4 - Cauterizing the epithelium of the lumen of the prostatic segment of the vas.
6. Completely transect the vas using scissors or the heated cautery device (Figure-5).

a. Tip: Complete the cut exactly at the site that has been hemi-transected.

b. Tip: Do not apply traction on the ring forceps -the testicular stump- while cutting the vas. Traction may cause the prostatic stump to slide too deeply into the scrotum.

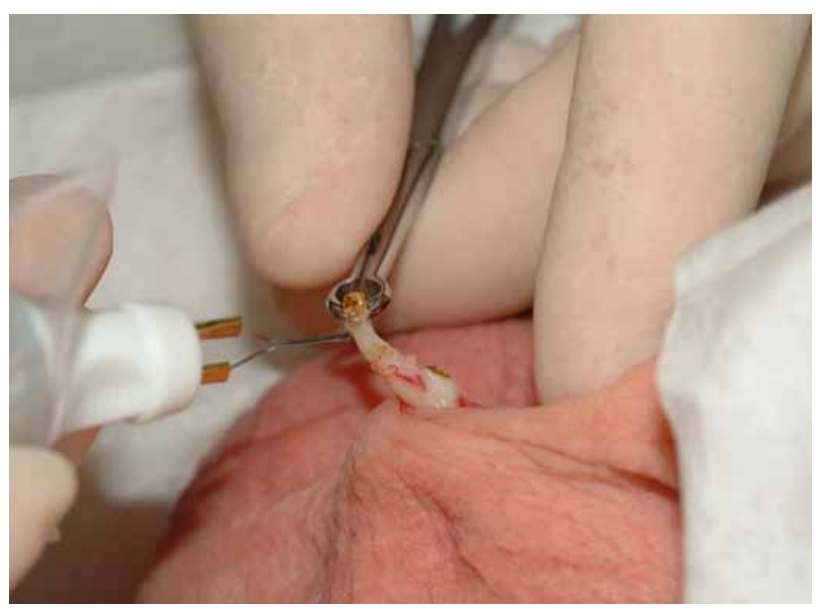

Figure 5 - Cutting the vas with a thermal cautery device.

7. To cover the prostatic stump, use the dissecting forceps or small tooth forceps (Adson) to grasp the full thickness of the sheath (internal spermatic fascia) at mid-distance between where the fascia sticks to the testicular vas segment and the prostatic stump (Figure-6).

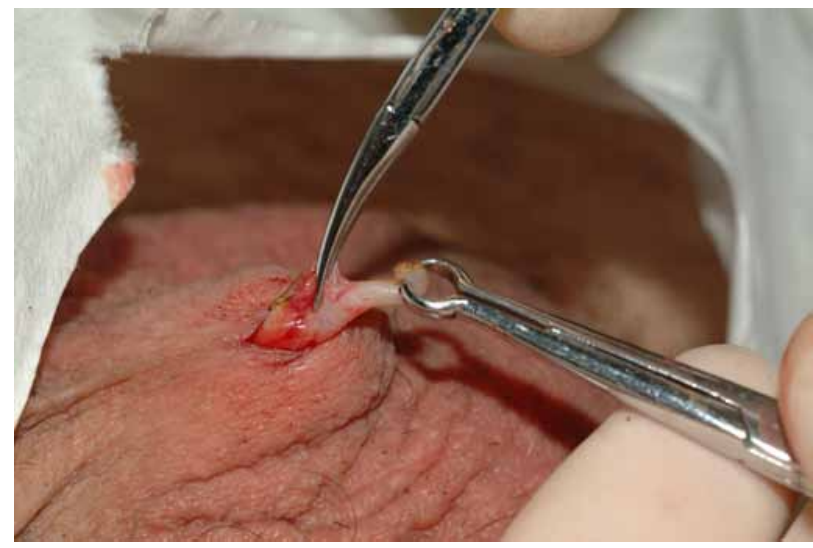

Figure 6 - Grasping the internal spermatic fascia with dissecting forceps. 
a. Tip: If the prostatic stump does not slide spontaneously into its sheath, do one of the following actions until the vas slides into its sheath:

i. Make sure the posterior wall of the vas (including its sheath) has been completely transected by very gently making a further cut with the cautery device. After adequate transaction, a loop will no longer be observed (Figure-5).

ii. Gently use the dissecting or tooth forceps to push the prostatic stump into its sheath.

iii. Pinch the fascia over the stump with thumb and index finger.

b. Tip: When grasping the fascia, make sure to grasp both sides of the sheath in order to cover the prostatic stump.

c. Tip: Grasping the fascia too high or too low prevents adequate full coverage of the stump.

8. Holding the fascia firmly with the dissecting forceps or the tooth forceps, gently pull the testicular end away in order to separate 2-3 mm of the fascia covering the testicular segment (Figure-7). This step is essential to ligating a portion of the fascia that covers the testicular stump along with the fascia that covers the prostatic stump and thereby performing adequate FI (see Step 9).

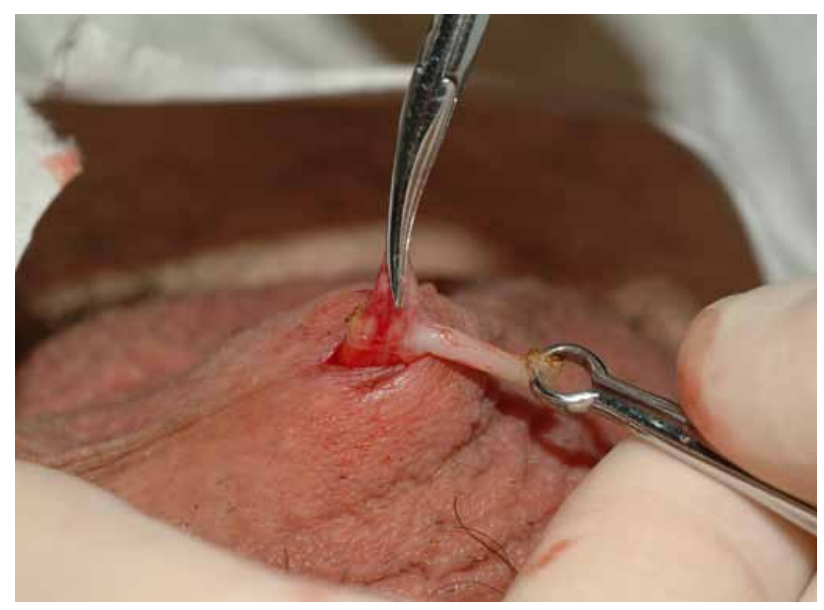

Figure 7 - Pulling on the testicular stump to completely separate the sheath covering the testicular stump.
9. Put a 2-0 or 3-0 silk (or other suture material) free tie with at least 3 knots on the fascia overlying the prostatic stump (Figures 8 and 9).

a. Tip: Make sure that a portion of the fascia covering the testicular segment is ligated over the prostatic stump.

b. Tip: Vessels may be ligated concomitantly to control bleeding.

c. Tip: Make sure that only the fascia and not the prostatic stump is ligated.

d. Tip: Ligation of the fascia may help to completely push the prostatic stump into the fascia if this was not adequately achieved in Step 7: see Tip a.

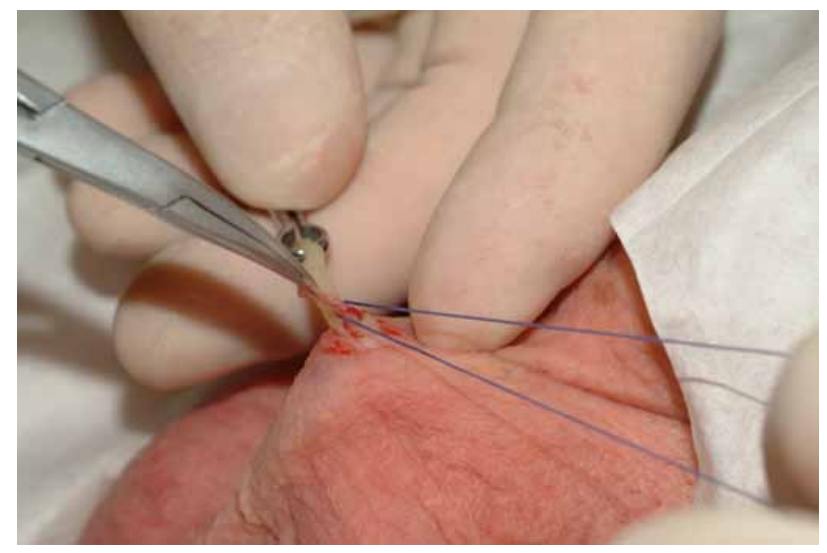

Figure 8 - Ligating the fascia over the prostatic stump (1).

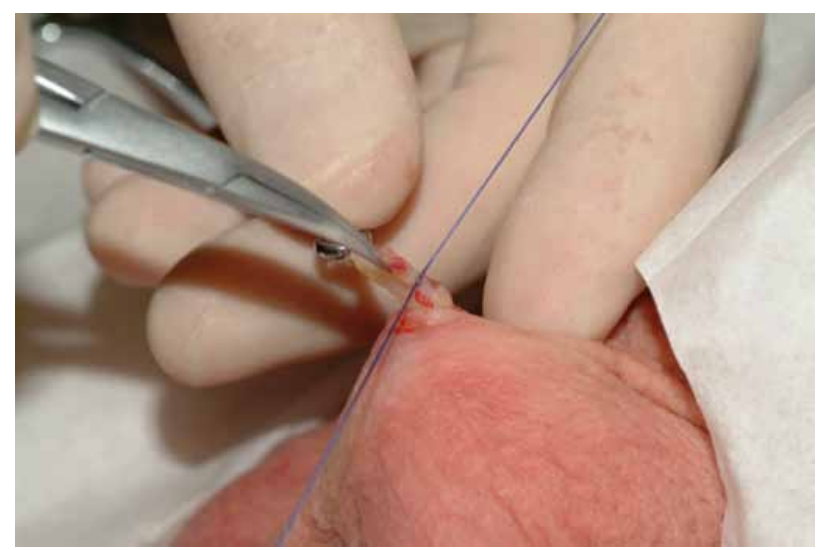

Figure 9 - Ligating the fascia over the prostatic stump (2).

10. Cut the threads.

11. Hemi-transect the testicular end of the vas to leave a segment of about 0.5 to $1 \mathrm{~cm}$ grasped into the ringed clamp. This segment will be excised. 
12. Repeat Steps 4 to 6 on the testicular end (Figure-10).

a. Tip: Before excising the vas segment and letting the prostatic and testicular vas stumps slip into the scrotum, check carefully for bleeding.

i. Use the cautery device to control minor bleeding.

ii. A suture over the fascia at the base of the testicular segment may control persistent bleeding.

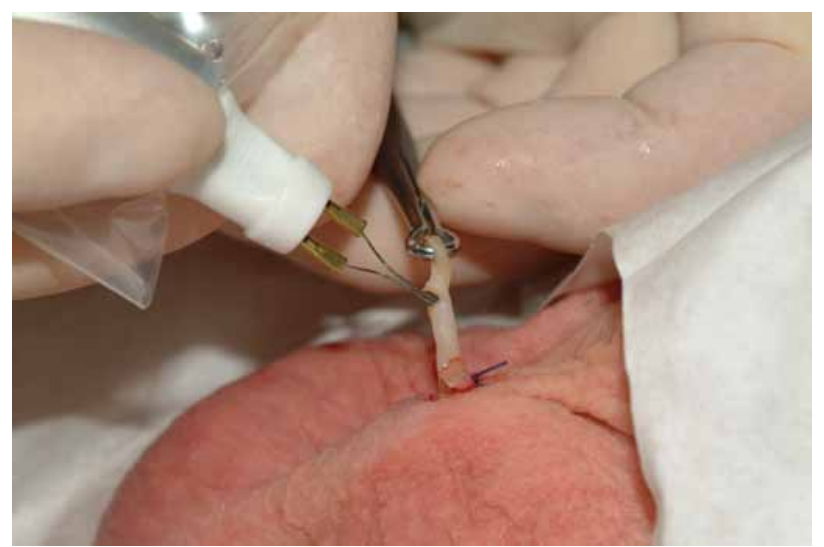

Figure 10 - Cauterizing the lumen of the testicular stump after completing FI on the prostatic stump.

Note: The testicular end may be left open ("open-end vasectomy") by completely transecting the vas with scissors at Step 11 and omitting Step 12. In this case, check carefully for any bleeding before cutting the vas and letting it slip into the scrotum.

13. Repeat the procedure on the contralateral vas.

\section{COMMENTS}

A video of this surgical technique may be viewed at www.youtube.com/ watch? $\mathrm{v}=\mathrm{Pw} 80 \mathrm{QNbnVig}$. Pilot assessments in many countries in Asia (5), in Rwanda, and in Canada showed that this technique can be safely and effectively performed by new vasectomy providers and surgeons who already perform vasectomies. Nevertheless, there may be barriers to implementing this technique on a large scale in low-resources settings. Direct costs (cautery devices, tips, and batteries) and indirect costs (training, processing, and the mainte- nance of the devices) would need to be considered. However, the Program for Appropriate Technology in Health (PATH) has determined that cautery handles and tips currently available in the United States and Canada are durable and can be safely reused, and that the proposed technique would be cost-effective in various low-resource settings (6). For this reason, new vasectomy providers and surgeons who already perform vasectomies using LE should consider learning thermal cautery and FI to occlude vas deferens in order to maximize the effectiveness of the procedure.

\section{CONFLICT OF INTEREST}

None declared.

\section{ACKNOWLEDGMENT}

We wish to thanks Mrs. Jennifer Petrela for reviewing the manuscript.

\section{REFERENCES}

1. Labrecque M, Dufresne C, Barone MA, St-Hilaire K: Vasectomy surgical techniques: a systematic review. BMC Med. 2004; 2:21.

2. Sokal DC, Labrecque M: Effectiveness of vasectomy techniques. Urol Clin North Am. 2009; 36: 317-29.

3. Aradhya KW, Best K, Sokal DC: Recent developments in vasectomy. BMJ. 2005; 330: 296-9.

4. Barone MA, Hutchinson PL, Johnson CH, Hsia J, Wheeler J: Vasectomy in the United States, 2002. J Urol. 2006; 176: 232-6; discussion 236.

5. Labrecque M, Pile J, Sokal D, Kaza RC, Rahman M, Bodh SS, et al.: Vasectomy surgical techniques in South and South East Asia. BMC Urol. 2005; 5: 10.

6. Seamans Y, Harner-Jay CM: Modelling cost-effectiveness of different vasectomy methods in India, Kenya, and Mexico. Cost Eff Resour Alloc. 2007; 5: 8.

7. No-Scalpel Vasectomy. An Illustrated Guide for Surgeons. Third edition. New York, NY. EngenderHealth. 2003; pp. 1-59.

8. Moss WM: Sutureless vasectomy, an improved technique: 1300 cases performed without failure. Fertil Steril. 1976; 27: 1040-5. 
9. Moss WM: A comparison of open-end versus closedend vasectomies: a report on 6220 cases. Contraception. 1992; 46: 521-5.

10. Labrecque M, Nazerali H, Mondor M, Fortin V, Nasution M: Effectiveness and complications associated with 2 vasectomy occlusion techniques. J Urol. 2002; 168: 2495-8; discussion 2498.
11. Reynolds RD: Vas deferens occlusion during no-scalpel vasectomy. J Fam Pract. 1994; 39: 577-82.

Submitted for publication: January 07, 2011

Accepted after revision: May 13, 2011

\section{Correspondence address:}

Dr. Michel Labrecque

Hôpital Saint-François d'Assise, D6-728, 10 rue de l'Espinay,

Québec, Canada, G1L 3L5

Fax: + 418 525-4194

E-mail: michel.labrecque@mfa.ulaval.ca 